\title{
NF-kappaB inducing kinase (NIK) is a key regulator of inflammation-induced angiogenesis
}

\author{
Ae-Ri Noort ${ }^{1}$, Katinka PM van Zoest ${ }^{1}$, Pieter Koolwijk², Paul-Peter Tak', Sander W Tas ${ }^{1 *}$ \\ From 7th European Workshop on Immune-Mediated Inflammatory Diseases \\ Noordwijk aan Zee, the Netherlands. 28-30 November 2012
}

\section{Background}

In rheumatoid arthritis (RA) synovial tissue (ST) angiogenesis can be observed already in the earliest phase of disease. The chemokine CXCL12, which is induced via the non-canonical nuclear factor-kappaB (NF-kB) pathway, plays an important role in angiogenesis, lymphocyte transendothelial migration, and the homing of endothelial progenitor cells. Therefore, the non-canonical pathway, with its key mediator NF-kB inducing kinase (NIK), may play an important role in pathological angiogenesis and the perpetuation of synovial inflammation in RA.

\section{Objective}

To study the role of non-canonical NF-kB signaling in pathological angiogenesis in RA.

\section{Methods}

Expression of NIK and CXCL12 in RA ST was evaluated using immunofluorescence microscopy (IF). Angiogenesis was studied in endothelial cells (EC) in vitro using the tube formation assay and ex vivo by comparing WT and $\mathrm{NIK}^{-1-}$ mice in the aortic ring assay. Physiological (developmental) angiogenesis was evaluated by isolectin B4 staining of the retina followed by confocal microscopy. The contribution of NIK to synovial angiogenesis was studied in vivo in antigen-induced arthritis (AIA).

\section{Results}

NIK, p52 and CXCL12 were highly expressed in EC in RA ST, mainly in small (newly formed) blood vessels. Stimuli that induce non-canonical NF-kB signaling (lymphotoxin (LT), LIGHT, and CD40L) significantly enhanced in vitro tube formation 2,5-fold $(\mathrm{p}<0.05)$, which could be completely blocked by siRNA targeting NIK or IKK $\alpha$. Aortic

'Division of Clinical Immunology \& Rheumatology, Academic Medical Center, University of Amsterdam, The Netherlands

Full list of author information is available at the end of the article rings from WT and $\mathrm{NIK}^{-1-}$ mice showed normal TNF- and VEGF-induced microvessel outgrowth. In contrast, whereas non-canonical NF-kB stimuli induced microvessel outgrowth in WT mice (unstim $29.94 \pm 6.08$ vs. LT $159.1 \pm 50.24$ vs. LIGHT $\left.110.3 \pm 17.68\left(\mathrm{~mm}^{\wedge} 2\right) \mathrm{p}<0.05\right)$, no microvessel outgrowth was observed in aortic rings from $\mathrm{NIK}^{-1-}$ mice (unstim $28.74 \pm 15.89$ vs. LT $45.9 \pm$ 16.71 vs. LIGHT $\left.43.41 \pm 15.73\left(\mathrm{~mm}^{\wedge} 2\right)\right)$. In line with this, $\mathrm{NIK}^{-1-}$ mice exhibited normal developmental angiogenesis in the retina, but a $50 \%$ reduction in pathological angiogenesis in synovial inflammation (blood vessels in synovial tissue WT $20 \pm 5.07$ vs. $\mathrm{NIK}^{-1-} 10.2 \pm 3.02$ ).

\section{Conclusions}

NIK is preferentially expressed in EC in RA ST and noncanonical NF-kB signaling in EC results in enhanced angiogenesis in vitro. $\mathrm{NIK}^{-1-}$ mice exhibited normal developmental and VEGF-induced angiogenesis, but reduced pathological angiogenesis in AIA. These findings point towards an important role of the non-canonical NF-kB pathway in pathological angiogenesis associated with chronic (synovial) inflammation. This could be exploited for the development of future new therapies for RA.

\section{Author details}

'Division of Clinical Immunology \& Rheumatology, Academic Medical Center, University of Amsterdam, The Netherlands. ${ }^{2}$ Dept. of Physiology, Institute for Cardiovascular Research (ICaR-VU), VU University Medical Center, Amsterdam, The Netherlands.

Published: 28 November 2012

doi:10.1186/1479-5876-10-S3-01

Cite this article as: Noort et al:: NF-kappaB inducing kinase (NIK) is a key regulator of inflammation-induced angiogenesis. Journal of Translational Medicine 2012 10(Suppl 3):O1. 